\title{
Turing's Diffusive Threshold in Random Reaction-Diffusion Systems
}

\author{
Pierre A. Haas $\odot^{*}$ \\ Mathematical Institute, University of Oxford, Woodstock Road, Oxford OX2 6GG, United Kingdom \\ Raymond E. Goldstein $\odot^{\dagger}$ \\ Department of Applied Mathematics and Theoretical Physics, Centre for Mathematical Sciences, \\ University of Cambridge, Wilberforce Road, Cambridge CB3 OWA, United Kingdom
}

(Received 9 November 2020; accepted 29 April 2021; published 9 June 2021)

\begin{abstract}
Turing instabilities of reaction-diffusion systems can only arise if the diffusivities of the chemical species are sufficiently different. This threshold is unphysical in most systems with $N=2$ diffusing species, forcing experimental realizations of the instability to rely on fluctuations or additional nondiffusing species. Here, we ask whether this diffusive threshold lowers for $N>2$ to allow "true" Turing instabilities. Inspired by May's analysis of the stability of random ecological communities, we analyze the probability distribution of the diffusive threshold in reaction-diffusion systems defined by random matrices describing linearized dynamics near a homogeneous fixed point. In the numerically tractable cases $N \leqslant 6$, we find that the diffusive threshold becomes more likely to be smaller and physical as $N$ increases, and that most of these many-species instabilities cannot be described by reduced models with fewer diffusing species.
\end{abstract}

DOI: 10.1103/PhysRevLett.126.238101

In 1952, Turing described the pattern-forming instability that now bears his name [1]: diffusion can destabilize a fixed point of a system of reactions that is stable in wellmixed conditions. Nigh on threescore and ten years on, the contribution of Turing's mechanism to chemical and biological morphogenesis remains debated, not least because of the diffusive threshold inherent in the mechanism: chemical species in reaction systems are expected to have roughly equal diffusivities, yet Turing instabilities cannot arise at equal diffusivities [2,3]. It remains an open problem to determine the diffusivity difference required for generic systems to undergo this instability, yet this diffusive threshold has been recognized at least since reduced models of the Belousov-Zhabotinsky reaction [4,5] only produced Turing patterns at unphysically large diffusivity differences.

Therefore, the first experimental realizations of Turing instabilities [6-8] relied on gel reactors in which reversible binding to the (nondiffusing) substrate greatly reduced the effective diffusivity of one species $[9,10]$. A biological analog is membrane binding in protein pattern formation [11]. (Additionally, transmembrane transport in biological tissues can increase effective diffusivity differences [12].) More abstractly, a third, nondiffusing species can allow

Published by the American Physical Society under the terms of the Creative Commons Attribution 4.0 International license. Further distribution of this work must maintain attribution to the author(s) and the published article's title, journal citation, and DOI.
Turing instabilities even if the $N=2$ diffusing species have equal diffusivities [13-15]. Such nondiffusing species continue to permeate recent work on the network topology of Turing systems [16,17].

Moreover, fluctuation-driven instabilities in reactiondiffusion systems have noise-amplifying properties that allow their pattern amplitude to be comparable to that of deterministic Turing patterns [18], with a lower diffusive threshold than the deterministic one [19-22]. A synthetic bacterial population with $N=2$ species that exhibits patterns in agreement with such a stochastic instability, but does not satisfy the conditions for a deterministic instability [23], was reported recently.

These experimental instabilities relying on fluctuations or additional nondiffusing species and the nonlinear instabilities arising from finite-amplitude perturbations [2] are different from the true Turing instabilities defined by Turing [1]. Can such instabilities be realized, instead, in systems with $N>2$ diffusing species? Equivalently, is the diffusive threshold lower in such systems? These questions have remained unanswered, perhaps because, in contrast to the textbook case $N=2$ and the concomitant picture of an "inhibitor" out-diffusing an "activator" [24,25], the complicated instability conditions for $N>2$ [26] do not lend themselves to analytical progress.

Here, we analyze the diffusive threshold for Turing instabilities with $2 \leqslant N \leqslant 6$ diffusing species. Inspired by May's work on random ecological communities [27], we analyze random Turing instabilities by sampling random matrices that represent the linearized reaction dynamics of otherwise unspecified reaction-diffusion 
systems. A semianalytic approach shows that the diffusive threshold is more likely to be smaller and physical for $N=3$ compared to $N=2$, and that two of the three diffusivities are equal at the transition to instability. We extend these results to the remaining numerically tractable cases of reaction-diffusion systems with $4 \leqslant N \leqslant 6$ and two different diffusivities: their Turing instabilities are still more likely to have a smaller and physical diffusive threshold, but most of them cannot be described by reduced models with fewer diffusing species.

We begin with the simplest case, $N=2$, in which concentrations $u, v$ obey

$$
\dot{u}=f(u, v)+d_{u} \nabla^{2} u, \quad \dot{v}=g(u, v)+d_{v} \nabla^{2} v .
$$

The conditions for Turing instability in this system [25] only depend on the four entries of the Jacobian

$$
\mathbf{J}=\left(\begin{array}{ll}
f_{u} & f_{v} \\
g_{u} & g_{v}
\end{array}\right)
$$

the partial derivatives of the reaction system at a fixed point $\left(u_{*}, v_{*}\right)$ of the homogeneous system. This fixed point is stable to homogeneous perturbations if and only if $J \equiv \operatorname{det} J>0$ and $I_{1} \equiv \operatorname{tr} J<0$. A stable fixed point of this kind is unstable to a Turing instability only if $p \equiv-f_{u} g_{v}>0$ [25]. Defining the diffusion coefficient ratio $D_{2}=\max \left\{d_{u} / d_{v}, d_{v} / d_{u}\right\} \geqslant 1$, a Turing instability occurs if and only if these conditions hold along with [28]

$$
D_{2} \geqslant D_{2}^{*} \equiv\left(\frac{\sqrt{J}+\sqrt{J+p}}{\min \left\{\left|f_{u}\right|,\left|g_{v}\right|\right\}}\right)^{2} .
$$

This diffusivity difference $D_{2}^{*}$, which is required mathematically for instability, is unphysical [Fig. 1(a)] if it exceeds the diffusivity difference $\mathcal{D} \geqslant 1$ of the physical system: $\mathcal{D} \approx 1$ for similarly sized molecules in solution, but, e.g., $\mathcal{D} \approx 20$ for the stochastic Turing instability observed in Ref. [23]. Hereinafter, we take $\mathcal{D}=5$ arbitrarily in numerical examples (but have checked that the value of $\mathcal{D}$ does not affect results qualitatively). To quantify $D_{2}^{*}$, we introduce the range $R$ of kinetic parameters,

$$
R \equiv \frac{\max \left\{\left|f_{u}\right|,\left|f_{v}\right|,\left|g_{u}\right|,\left|g_{v}\right|\right\}}{\min \left\{\left|f_{u}\right|,\left|f_{v}\right|,\left|g_{u}\right|,\left|g_{v}\right|\right\}} .
$$

If $g(u, v)=-f(u, v)$, Eqs. (1) describe a massconserving reaction-diffusion system [29]. For such a system [28], $D_{2}^{*}=R$, and so the instability is physical if and only if $R<\mathcal{D}$. However, while $\mathcal{D}$ is expected to be "small" in many cases, there is no physical reason to expect $R$ to be similarly small. Therefore, the condition $R<\mathcal{D}$ expresses a fine-tuning problem for the reaction kinetics.

We now return to general systems, for which one obtains [28] the upper bound
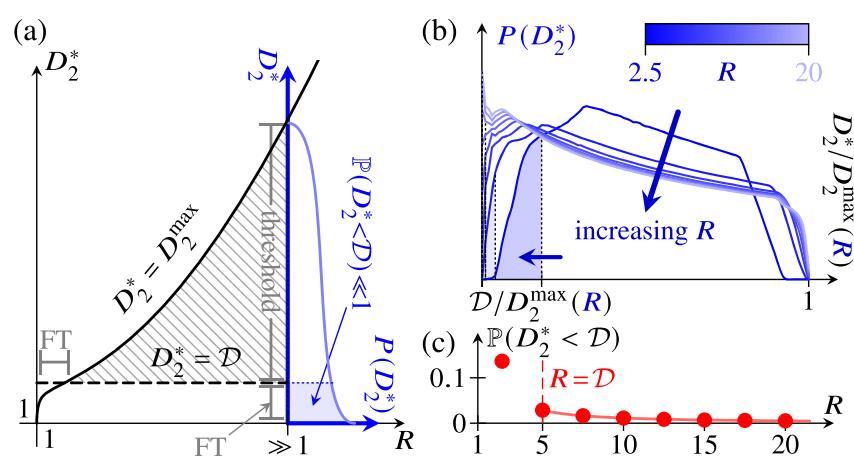

FIG. 1. Turing's diffusive threshold for $N=2$. (a) Cartoon of the diffusive threshold and the fine-tuning $(\mathrm{FT})$ problem for $R \approx 1$ and $R \gg 1$. The diffusivity difference required mathematically is unphysical in the hatched region $\mathcal{D} \leqslant D_{2}^{*} \leqslant D_{2}^{\max }$. (b) Distribution $P\left(D_{2}^{*}\right)$, supported on the (scaled) interval $\left[1, D_{2}^{\max }(R)\right]$, estimated for different $R$. (c) Plot of $\mathbb{P}\left(D_{2}^{*}<\mathcal{D}\right)$ [shaded areas in panels (a) and (b)] against $R$, revealing the diffusive threshold. Markers: estimates from panel (b); solid line: exact result [28] for $R>\mathcal{D}[35]$.

$$
D_{2}^{*} \leqslant D_{2}^{\max }(R) \equiv\left(R+\sqrt{R^{2}-1}\right)^{2}
$$

As $R \rightarrow 1, D_{2}^{\max } \rightarrow 1$ : there is no diffusive threshold, but this limit requires fine-tuning the reaction kinetics [Fig. 1(a)]. (In fact, it is a particular instance of singular kinetics that allow Turing instabilities at nearly equal diffusivities more generally [3].) If $R \gg 1$, then $D_{2}^{\max }=O\left(R^{2}\right)$. This does not imply the existence of a threshold, for it does not preclude most systems with range $R$ having $D_{2}^{*} \ll D_{2}^{\max }$ [Fig. 1(a)]. Therefore, the existence of a diffusive threshold relates to the distribution of $D_{2}^{*}$ for systems with range $R$.

To understand this distribution, we draw inspiration from May's statistical analysis of the stability of ecological communities [27], which studies random Jacobians, corresponding to equilibria of otherwise unspecified population dynamics. By analogy, we study random Turing instabilities, sampling uniformly and independently random Jacobians corresponding to equilibria of otherwise unspecified reaction kinetics, and analyze the criteria for them to be Turing unstable. There is, of course, no more reason to expect the kinetic parameters to be independent or uniformly distributed than there is reason to expect the linearized population dynamics in May's analysis [27] to be independent or normally distributed. Yet, in the absence of experimental understanding of what these parameter distributions should be (in either context), the potential of the random matrix approach to reveal stability principles has been amply demonstrated in population dynamics [36-46].

We sample the kinetic parameters in Eq. (2) independently and uniformly from $[-R,-1] \cup[1, R]$, randomly set one of them equal to \pm 1 and one equal to $\pm R$, and, thus, estimate the probability distribution $P\left(D_{2}^{*}\right)$ for fixed $R$ 
[Fig. 1(b)]. The threshold is quantified by the probability of a Turing instability being physical,

$$
\mathbb{P}\left(D_{2}^{*}<\mathcal{D}\right)=\int_{1}^{\mathcal{D}} P\left(D_{2}^{*}\right) d D_{2}^{*}
$$

Both from the estimates in Fig. 1(b) and by evaluating the integral in closed form [28], we find that $\mathbb{P}\left(D_{2}^{*}<\mathcal{D}\right)$ is tiny [Fig. 1(c)], except if $R<\mathcal{D}$. This generalizes the result for mass-conserving systems, and raises the fine-tuning problem again. The consequent rareness of physical Turing instabilities expresses the diffusive threshold for $N=2$.

To investigate how this threshold changes with $N$, next, we consider $N=3$ and

$$
\begin{gathered}
\dot{u}=f(u, v, w)+d_{u} \nabla^{2} u, \\
\dot{v}=g(u, v, w)+d_{v} \nabla^{2} v, \\
\dot{w}=h(u, v, w)+\nabla^{2} w,
\end{gathered}
$$

where we have rescaled space to set $d_{w}=1$. We introduce the matrix of diffusivities and the reaction Jacobian,

$\mathbf{D}=\left(\begin{array}{ccc}d_{u} & 0 & 0 \\ 0 & d_{v} & 0 \\ 0 & 0 & 1\end{array}\right), \quad \mathbf{J}=\left(\begin{array}{ccc}f_{u} & f_{v} & f_{w} \\ g_{u} & g_{v} & g_{w} \\ h_{u} & h_{v} & h_{w}\end{array}\right)$,

in which the entries of $\mathbf{J}$ are again partial derivatives at a fixed point $\left(u_{*}, v_{*}, w_{*}\right)$ of the homogeneous system. This fixed point is unstable to a Turing instability if it is stable but, for some eigenvalue $-k^{2}<0$ of the Laplacian, $\overline{\mathrm{J}}\left(k^{2}\right)=\mathrm{J}-k^{2} \mathrm{D}$ is unstable [3], i.e., has an eigenvalue $\lambda$ such that $\operatorname{Re}(\lambda)<0$. More precisely, a Turing instability arises when a real eigenvalue of $\overline{\mathrm{J}}\left(k^{2}\right)$ crosses zero, i.e., when $\mathcal{J}\left(k^{2}\right) \equiv \operatorname{det} \overline{\mathrm{J}}\left(k^{2}\right)=0$ and, therefore, arises first at a wave number $k=k_{*}$ with $\mathcal{J}\left(k_{*}^{2}\right)=\partial \mathcal{J} / \partial k^{2}\left(k_{*}^{2}\right)=0$ [3]. Hence, $\mathcal{J}$, a cubic polynomial in $k^{2}$, has a double root at $k^{2}=k_{*}^{2}>0$, so its discriminant [31] vanishes. This discriminant, $\Delta\left(d_{u}, d_{v}\right)$, is a polynomial in $d_{u}, d_{v}$. We denote by $K\left(d_{u}, d_{v}\right)$ the double root of $\mathcal{J}$ corresponding to a point $\left(d_{u}, d_{v}\right)$ on the curve $\Delta\left(d_{u}, d_{v}\right)=0$.

Thus, determining the diffusive threshold for Turing instability in Eqs. (7) requires solving the problem

minimize $D_{3}\left(d_{u}, d_{v}\right)$ subject to $\left\{\begin{array}{l}\Delta\left(d_{u}, d_{v}\right)=0, \\ K\left(d_{u}, d_{v}\right)>0\end{array}\right.$

in which the diffusion coefficient ratio is

$$
D_{3}\left(d_{u}, d_{v}\right)=\max \left\{d_{u}, 1 / d_{u}, d_{v}, 1 / d_{v}, d_{u} / d_{v}, d_{v} / d_{u}\right\}
$$
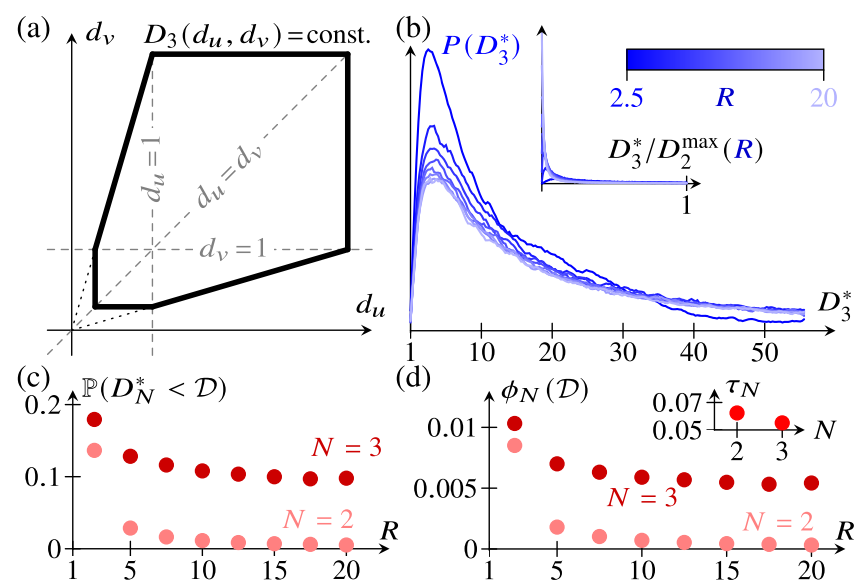

FIG. 2. Results for $N=3$. (a) Contours of $D_{3}\left(d_{u}, d_{v}\right)$ in the positive $\left(d_{u}, d_{v}\right)$ quadrant. (b) Smoothed distribution $P\left(D_{3}^{*}\right)$, estimated for different $R$. Inset: same plot, scaled to $\left[1, D_{2}^{\max }(R)\right]$ for comparison to $N=2$ in Fig. 1 (a). (c) $\mathbb{P}\left(D_{N}^{*}<\mathcal{D}\right)$ against $R$ for $N \in\{2,3\}$ : the diffusive threshold lowers for $N=3$ compared to $N=2$. (d) Proportion $\phi_{N}(\mathcal{D})$ of random Jacobians that have a physical Turing instability, plotted against $R$, for $N \in\{2,3\}$. Inset: proportion $\tau_{N}$ of random Jacobians that have a (physical or unphysical) Turing instability, averaged over $R$, for $N \in\{2,3\}[35]$.

With the aim of obtaining statistics for the minimal value $D_{3}^{*}$, direct numerical solution of this constrained optimization problem is obviously not a feasible approach. In the Supplemental Material [28], we therefore show how solving problem (9) reduces to polynomial root finding. This semianalytic approach reveals a particular class of minima, attained at the vertices of the contours of $D_{3}\left(d_{u}, d_{v}\right)$ [Fig. 2(a)], i.e., at $d_{u}=1, d_{v}=1$, or $d_{u}=d_{v}$. In these cases, $\Delta\left(d_{u}, d_{v}\right)=0$ is a (sextic) polynomial in a single variable. We call these minima "binary," since the corresponding systems have only two different diffusivities. We implement this approach numerically [28], and sample random systems similarly to the case $N=2$, drawing the entries of $\mathrm{J}$ in Eq. (8) uniformly and independently at fixed range $R$.

Remarkably, all global minima we found numerically were binary [28]. Thus, the minimizing systems come in two flavors: those with two "fast" diffusers and one "slow" diffuser, and those with one fast diffuser and two slow diffusers. Systems with a nondiffusing species are a limit of the former; this will be discussed below. The latter arise in models of scale pattern formation in fish and lizards $[47,48]$, in which short-range pigments respectively activate and inhibit a long-range factor.

The distribution of $D_{3}^{*}$ [Fig. 2(b)] has a different shape from that of $D_{2}^{*}$ [Figs. 1(a) and 2(b), inset]. While the support of the distribution of $D_{3}^{*}$ appears unbounded, Fig. 2(c) shows that $\mathbb{P}\left(D_{3}^{*}<\mathcal{D}\right)>\mathbb{P}\left(D_{2}^{*}<\mathcal{D}\right)$. Hence, the diffusivity difference is more likely to be physical for $N=3$ than for $N=2$ : the diffusive threshold is lowered. 
The proportion $\tau_{N}$ of random kinetic Jacobians that have a Turing instability (be it physical or unphysical) is smaller for $N=3$ than for $N=2$ [Fig. 2(d), inset]. This is not surprising, because a random Jacobian is less likely to correspond to a stable fixed point (which, we recall, is a necessary condition for Turing instability) for $N=3$ than for $N=2$, essentially because it has to satisfy more conditions for stability if $N=3$. It is therefore striking that the threshold reduces sufficiently for $N=3$ for the proportion $\phi_{N}(\mathcal{D})=\tau_{N} \mathbb{P}\left(D_{N}^{*}<\mathcal{D}\right)$ of random Jacobians with a physical Turing instability to be larger for $N=3$ than for $N=2$ [Fig. 2(d)], even though a Turing instability of any kind is more likely if $N=2$.

To extend these results to $N>3$ diffusing species, we consider the (linearized) reaction-diffusion system

$$
\dot{\boldsymbol{u}}=\mathrm{J} \cdot \boldsymbol{u}+\mathrm{D} \cdot \nabla^{2} \boldsymbol{u},
$$

where $\mathrm{J}$ is a random kinetic Jacobian, and $\mathrm{D}$ is a diagonal matrix of diffusivities. Even with our semianalytic approach, this cannot be analyzed for general D: not even for $N=4$ were we able to obtain closed forms of the required polynomials. To make further progress, we therefore restrict to binary $\mathrm{D}$ in which the $N$ diffusivities take two different values only, since we showed above that $D_{3}^{*}$ is attained for such binary $\mathrm{D}$. As in the case $N=3$, this reduces the discriminant condition $\Delta(\mathrm{D})=0$ to polynomial equations in one variable that determine the minimum diffusivity difference $D_{N}^{*}$ for these binary Turing instabilities [28].

Figure 3(a) shows that the diffusive threshold lowers further for $4 \leqslant N \leqslant 6$ in these systems. Meanwhile, the fact that most stable random kinetic Jacobians undergo such a binary Turing instability [Fig. 3(b)] suggests that these provide a useful picture of the threshold. However, $\phi_{N}(\mathcal{D})$ decreases for $N \geqslant 4$ [Fig. 3(c)] because $\tau_{N}$ decreases [Fig. 3(c), inset]. Nonetheless, as both $\mathbb{P}\left(D_{N}^{*}<\mathcal{D}\right)$ and the proportion $\sigma_{N}$ of stable random Jacobians that are Turing unstable increase [Figs. 3(a) and 3(b)], so does the proportion of stable random Jacobians having a physical Turing instability.

How, then, to realize the true Turing instabilities defined by Turing [1] experimentally? Our analysis shows that the diffusive threshold is more likely to be physical the more species there are, but how to find an experimental Turing instability in the first place? Turing instabilities remain rare in random systems even as the number of species increases, but the above shows that this rareness mainly results from the rareness of stable equilibria in such systems. Hence, the proverbial search for the needle in a haystack might be avoidable by evolving biochemical systems that admit a stable equilibrium toward a true Turing instability.

This analysis does not, however, reveal whether these instabilities lead to patterns observable at the physical scale of the system. Analysis of the wave number at which the

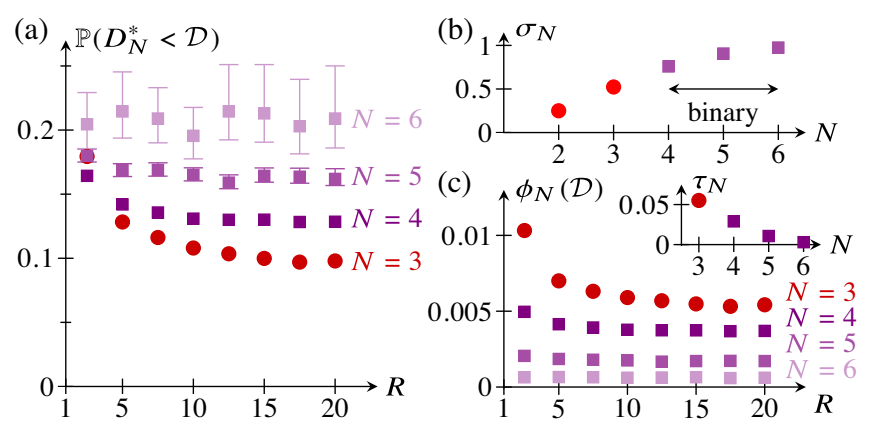

FIG. 3. Results for binary systems with $4 \leqslant N \leqslant 6$. (a) $\mathbb{P}\left(D_{N}^{*}<\mathcal{D}\right)$ against $R$ for $4 \leqslant N \leqslant 6$, revealing further lowering of the diffusive threshold compared to $N=3$. (b) Proportion $\sigma_{N}$ of random stable kinetic Jacobians that have a (binary, if $N>3$ ) Turing instability, averaged over $R$, and plotted against $N$. (c) Proportion $\phi_{N}(\mathcal{D})$ of random Jacobians that have a physical Turing instability plotted against $R$, for $3 \leqslant N \leqslant 6$. Inset: proportion $\tau_{N}$ of random Jacobians that have a (physical or unphysical) Turing instability, averaged over $R$, for $3 \leqslant N \leqslant 6$ [35].

linear instability first arises [28] suggests that we can extend our conclusions: Turing instabilities with more species are more likely to have physical diffusivity differences and to be observable. However, our statistical, linearized analysis cannot fully answer this question of observability, because its answer depends on the kinetic nonlinearities, which set the precise nature and scale of the Turing patterns that develop beyond onset of the instability; this is why we have relegated this analysis to the Supplemental Material [28].

The species in the binary systems with $3 \leqslant N \leqslant 6$ separate into fast and slow diffusers. The diffusion of these slow species is often ignored in analyses of systems of many chemical reactions [32], such as the full BelousovZhabotinsky reaction [49]. Corresponding reduced models are obtained by substituting the steady-state kinetics of the slow species into the remaining equations, thereby eliminating them [32]. The conditions for Turing instability in these reduced models are (almost) equivalent to those for the full model with nondiffusing slow species [32]. However, the diffusion of the slow species cannot in general be ignored: up to reordering species and rescaling space,

$$
\mathrm{D}=\left(\begin{array}{l|l}
\mathrm{I} & 0 \\
\hline 0 & d \mathrm{l}
\end{array}\right), \quad \mathrm{J}=\left(\begin{array}{l|l}
\mathrm{J}_{11} & \mathrm{~J}_{12} \\
\hline \mathrm{J}_{21} & \mathrm{~J}_{22}
\end{array}\right)
$$

where $d<1$ is the diffusivity of the slow diffusers. Reference [32] implies that there is a Turing instability with nondiffusing slow species $(d=0)$ only if $J_{11}-J_{12} J_{22}^{-1} J_{21}$ has a positive (real) eigenvalue [28]. Although the proportion of Turing unstable systems with $n \geqslant 2$ fast diffusers 

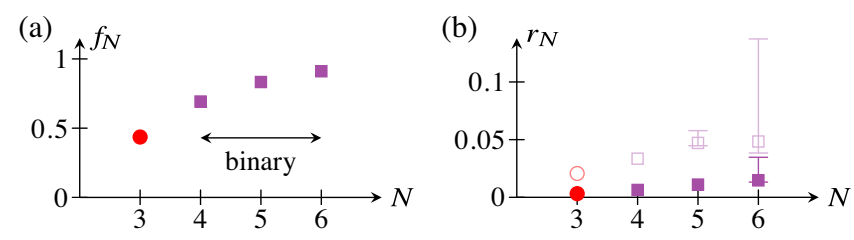

FIG. 4. Slow and fast diffusers in binary Turing instabilities with $3 \leqslant N \leqslant 6$. (a) Proportion $f_{N}$ of Turing unstable systems with $n \geqslant 2$ fast diffusers plotted against $N$, averaged over $R$. (b) Proportion $r_{N}$ of systems that remain Turing unstable at $d=0$, plotted against $N$, averaged over $R$. Filled markers: all Turing unstable systems; unfilled markers: physical Turing unstable systems with $D_{N}^{*}<\mathcal{D}$ [35].

(that could a priori still undergo a Turing instability with $d=0$ ) is large [Fig. 4(a)], the proportion of systems that do undergo such an instability is small, even if we restrict to those systems with physical diffusivity differences [Fig. 4(b)]. Hence, most of these binary Turing instabilities with $N>2$ species require all species to diffuse. Thus, they are more general than reduced instabilities with equal diffusivities and additional nondiffusing species. With our main result, that the diffusive threshold lowers as $N$ increases, this shows how these reduced models give but an incomplete picture of Turing instabilities.

In this Letter, we have analyzed random Turing instabilities to show how the diffusive threshold that has hampered experimental efforts to generate the true Turing instabilities defined by Turing [1] in systems of $N=2$ diffusing species lowers for systems with $N \geqslant 3$, most of whose instabilities cannot be described by reduced models with fewer diffusing species. However, why the threshold should be "large" in the first place remains unclear; this does not follow from the requirement of unequal diffusivities [2,3]. In this context, we prove an asymptotic result in the Supplemental Material [28]: for a Jacobian $\mathrm{J}$ to allow a Turing instability at almost equal diffusivities $D \approx I$, J must be even closer to a singular matrix $J_{0}$, i.e., $J-J_{0} \ll D-I$. Thus, the threshold $D-I$ is asymptotically large. Understanding how a large threshold arises more generally outside this asymptotic regime and lowers as $N$ increases remains an open problem, as do extending the present analysis to include the nonlocal interactions $[50,51]$ that arise, for example, in vegetation patterns [52] and extending previous work $[17,53]$ on the robustness of Turing patterns to $N \geqslant 3$. The latter, in particular, may help to identify those chemical or biological systems with $N \geqslant 3$ in which the true Turing instabilities discussed here can be realized experimentally.

We thank N. Goldenfeld, A. Krause, and P. K. Maini for discussions. This work was supported in part by a Nevile Research Fellowship from Magdalene College, Cambridge, and a Hooke Research Fellowship at the Mathematical Institute, University of Oxford (P. A. H.), Established
Career Fellowship No. EP/M017982/1 from the Engineering and Physical Sciences Research Council and Grant No. 7523 from the Marine Microbiology Initiative of the Gordon and Betty Moore Foundation (R. E. G.).

*haas@pks.mpg.de

Present address: Max Planck Institute for the Physics of Complex Systems, Nöthnitzer Straße 38, 01187 Dresden, Germany.

r.e.goldstein@damtp.cam.ac.uk

[1] A. M. Turing, The chemical basis of morphogenesis, Phil. Trans. R. Soc. B 237, 37 (1952).

[2] J. A. Vastano, J.E. Pearson, W. Horsthemke, and H. L. Swinney, Chemical pattern formation with equal diffusion coefficients, Phys. Lett. A 124, 320 (1987).

[3] J. E. Pearson and W. Horsthemke, Turing instabilities with nearly equal diffusion coefficients, J. Chem. Phys. 90, 1588 (1989).

[4] P. K. Becker and R. J. Field, Stationary concentration patterns in the Oregonator model of the BelousovZhabotinskii reaction, J. Phys. Chem. 89, 118 (1985).

[5] A. B. Rovinskii, Turing bifurcation and stationary patterns in the ferroin-catalyzed Belousov-Zhabotinskii reaction, J. Phys. Chem. 91, 4606 (1987).

[6] V. Castets, E. Dulos, J. Boissonade, and P. De Kepper, Experimental Evidence of a Sustained Standing TuringType Nonequilibrium Chemical Pattern, Phys. Rev. Lett. 64, 2953 (1990).

[7] P. De Kepper, V. Castets, E. Dulos, and J. Boissonade, Turing-type chemical patterns in the chlorite-iodide-malonic acid reaction, Physica (Amsterdam) 49D, 161 (1991).

[8] Q. Ouyang and H. L. Swinney, Transition from a uniform state to hexagonal and striped Turing patterns, Nature (London) 352, 610 (1991).

[9] I. Lengyel and I. R. Epstein, Modeling of Turing structures in the chlorite-iodide-malonic acid-starch reaction system, Science 251, 650 (1991).

[10] E. Dulos, J. Boissonade, J. J. Perraud, B. Rudovics, and P. De Kepper, Chemical morphogenesis: Turing patterns in an experimental chemical system, Acta Biotheor. 44, 249 (1996).

[11] E. Frey, J. Halatek, S. Kretschmer, and P. Schwille, Protein pattern formation, in Physics of Biological Membranes, edited by P. Bassereau and P. Sens (Springer, Berlin, Germany, 2018), pp. 229-260.

[12] P. Recho, A. Hallou, and E. Hannezo, Theory of mechanochemical patterning in biphasic biological tissues, Proc. Natl. Acad. Sci. U.S.A. 116, 5344 (2019).

[13] I. Lengyel and I. R. Epstein, A chemical approach to designing Turing patterns in reaction-diffusion systems, Proc. Natl. Acad. Sci. U.S.A. 89, 3977 (1992).

[14] J.E. Pearson, Pattern formation in a $(2+1)$-species activator-inhibitor-immobilizer system, Physica (Amsterdam) 188A, 178 (1992).

[15] K. Korvasová, E. A. Gaffney, P. K. Maini, M. A. Ferreira, and V. Klika, Investigating the Turing conditions for diffusion-driven instability in the presence of a binding immobile substrate, J. Theor. Biol. 367, 286 (2015). 
[16] L. Marcon, X. Diego, J. Sharpe, and P. Müller, Highthroughput mathematical analysis identifies Turing networks for patterning with equally diffusing signals, eLife 5, e14022 (2016).

[17] X. Diego, L. Marcon, P. Müller, and J. Sharpe, Key Features of Turing Systems are Determined Purely by Network Topology, Phys. Rev. X 8, 021071 (2018).

[18] T. Biancalani, F. Jafarpour, and N. Goldenfeld, Giant Amplification of Noise in Fluctuation-Induced Pattern Formation, Phys. Rev. Lett. 118, 018101 (2017).

[19] T. Butler and N. Goldenfeld, Robust ecological pattern formation induced by demographic noise, Phys. Rev. E 80, 030902(R) (2009).

[20] T. Biancalani, D. Fanelli, and F. Di Patti, Stochastic Turing patterns in the Brusselator model, Phys. Rev. E 81, 046215 (2010).

[21] T. Butler and N. Goldenfeld, Fluctuation-driven Turing patterns, Phys. Rev. E 84, 011112 (2011).

[22] F. Di Patti, L. Lavacchi, R. Arbel-Goren, L. Schein-Lubomirsky, D. Fanelli, and J. Stavans, Robust stochastic Turing patterns in the development of a onedimensional cyanobacterial organism, PLoS Biol. 16, e2004877 (2018)

[23] D. Karig, K. M. Martini, T. Lu, N. A. DeLateur, N. Goldenfeld, and R. Weiss, Stochastic Turing patterns in a synthetic bacterial population, Proc. Natl. Acad. Sci. U.S.A. 115, 6572 (2018).

[24] A. J. Koch and H. Meinhardt, Biological pattern formation: From basic mechanisms to complex structures, Rev. Mod. Phys. 66, 1481 (1994).

[25] J. D. Murray, in Mathematical Biology (Springer, Berlin, Germany, 2002), Vol. I, Appendix B.1, pp. 507-509 and Vol. II, Chap. 2, pp. 71-140, 3rd ed.

[26] R. A. Satnoianu, M. Menzinger, and P. K. Maini, Turing instabilities in general systems, J. Math. Biol. 41, 493 (2000).

[27] R. M. May, Will a large complex ecosystem be stable? Nature (London) 238, 413 (1972).

[28] See Supplemental Material at http://link.aps.org/ supplemental/10.1103/PhysRevLett.126.238101, which includes Refs. [3,25,29-34], for details of calculations for $N=2$, the derivation of the semianalytic approach for $N>2$ and a discussion of its numerical implementation, statistics of the wave number of Turing instabilities, the derivation of results for "slow" species, the proof of an asymptotic result, and for PYTHON3 code.

[29] F. Brauns, J. Halatek, and E. Frey, Phase-Space Geometry of Mass-Conserving Reaction-Diffusion Dynamics, Phys. Rev. X 10, 041036 (2020).

[30] F. Johansson, MPMATH: a PYTHON Library for ArbitraryPrecision Floating-Point Arithmetic (version 1.1.0, 2018).

[31] C.-K. Yap, in Fundamental Problems in Algorithmic Algebra (Oxford University Press, Oxford, England, 2000), Chaps. 6 and 7, pp. 141-218.

[32] S. Smith and N. Dalchau, Model reduction enables Turing instability analysis of large reaction-diffusion models, J. R. Soc. Interface 15, 20170805 (2018).

[33] E. J. Hinch, in Perturbation Methods (Cambridge University Press, Cambridge, England, 1991), Chap. 1.6, pp. 15-18.
[34] V. B. Lidskii, Perturbation theory of non-conjugate operators, USSR Comput. Math. Math. Phys. 6, 73 (1966).

[35] All results shown are for $\mathcal{D}=5$. The asymmetric error bars in Figs. 3 and 4 show 95\% confidence intervals larger than the plot markers, corrected for systems for which the numerics failed.

[36] S. Allesina and S. Tang, Stability criteria for complex ecosystems, Nature (London) 483, 205 (2012).

[37] A. Mougi and M. Kondoh, Diversity of interaction types and ecological community stability, Science 337, 349 (2012).

[38] K. Z. Coyte, J. Schluter, and K. R. Foster, The ecology of the microbiome: Networks, competition, and stability, Science 350, 663 (2015).

[39] J. Grilli, T. Rogers, and S. Allesina, Modularity and stability in ecological communities, Nat. Commun. 7, 12031 (2016).

[40] T. Gibbs, J. Grilli, T. Rogers, and S. Allesina, Effect of population abundances on the stability of large random ecosystems, Phys. Rev. E 98, 022410 (2018).

[41] C. A. Serván, J. A. Capitán, J. Grilli, K. E. Morrison, and S. Allesina, Coexistence of many species in random ecosystems, Nat. Ecol. Evol. 2, 1237 (2018).

[42] S. Butler and J. P. O'Dwyer, Stability criteria for complex microbial communities, Nat. Commun. 9, 2970 (2018).

[43] L. Stone, The feasibility and stability of large complex biological networks: A random matrix approach, Sci. Rep. 8, 8246 (2018).

[44] D. S. Maynard, C. A. Serván, J. A. Capitán, and S. Allesina, Phenotypic variability promotes diversity and stability in competitive communities, Ecol. Lett. 22, 1776 (2019).

[45] P. A. Haas, N. M. Oliveira, and R. E. Goldstein, Subpopulations and stability in microbial communities, Phys. Rev. Research 2, 022036(R) (2020).

[46] J. W. Barron and T. Galla, Dispersal-induced instability in complex ecosystems, Nat. Commun. 11, 6032 (2020).

[47] A. Nakamasu, G. Takahashi, A. Kanbe, and S. Kondo, Interactions between zebrafish pigment cells responsible for the generation of Turing patterns, Proc. Natl. Acad. Sci. U.S.A. 106, 8429 (2009).

[48] L. Manukyan, S. A. Montandon, A. Fofonjka, S. Smirnov, and M.C. Milinkovitch, A living mesoscopic cellular automaton made of skin scales, Nature (London) 544, 173 (2017).

[49] L. Györgi, T. Turányi, and R. J. Field, Mechanistic details of the oscillator Belousov-Zhabotinskii reaction, J. Phys. Chem. 94, 7162 (1990).

[50] T. Ohta, M. Mimura, and R. Kobayashi, Higher-dimensional localized patterns in excitable media, Physica (Amsterdam) 34D, 115 (1989).

[51] R. E. Goldstein, D. J. Muraki, and D. M. Petrich, Interface proliferation and the growth of labyrinths in a reactiondiffusion system, Phys. Rev. E 53, 3933 (1996).

[52] E. Meron, Vegetation pattern formation: The mechanisms behind the forms, Phys. Today 72, No. 11, 30 (2019).

[53] N. S. Scholes, D. Schnoerr, M. Isalan, and M. P. H. Stumpf, A comprehensive network atlas reveals that Turing patterns are common but not robust, Cell Syst. 9, 243 (2019). 